\title{
Space-based Reconfigurable Software Defined Radio Test Bed aboard International Space Station
}

\author{
Richard C. Reinhart \\ National Aeronautical and Space Administration \\ John H. Glenn Research Center \\ Cleveland, Ohio 44135 \\ richard.c.reinhart@nasa.gov \\ James P. Lux \\ Jet Propulsion Laboratory \\ Pasadena, California 91109 \\ james.p.lux@jpl.nasa.gov
}

Abstract - The National Aeronautical and Space Administration (NASA) recently launched a new software defined radio research test bed to the International Space Station. The test bed, sponsored by the Space Communications and Navigation (SCaN) Office within NASA is referred to as the SCaN Testbed. The SCaN Testbed is a highly capable communications system, composed of three software defined radios, integrated into a flight system, and mounted to the truss of the International Space Station. Software defined radios offer the future promise of in-flight reconfigurability, autonomy, and eventually cognitive operation. The adoption of software defined radios offers space missions a new way to develop and operate space transceivers for communications and navigation. Reconfigurable or software defined radios with communications and navigation functions implemented in software or VHDL (Very High Speed Hardware Description Language) provide the capability to change the functionality of the radio during development or after launch. The ability to change the operating characteristics of a radio through software once deployed to space offers the flexibility to adapt to new science opportunities, recover from anomalies within the science payload or communication system, and potentially reduce development cost and risk by adapting generic space platforms to meet specific mission requirements. The software defined radios on the $\mathrm{SCaN}$ Testbed are each compliant to NASA's Space Telecommunications Radio System (STRS) Architecture. The STRS Architecture is an open, non-proprietary architecture that defines interfaces for the connections between radio components. It provides an operating environment to abstract the communication waveform application from the underlying platform specific hardware such as digital-to-analog converters, analog-to-digital converters, oscillators, RF attenuators, automatic gain control circuits, FPGAs, general-purpose processors, etc. and the interconnections among different radio components.

NASA is inviting experimenters from industry, academia, and other agencies to use the SCaN Testbed. On the flight system, the three software defined radios (SDR) and a portion of the flight computer or avionics, is available to experimenters to develop, test, and operate new SDR applications in-orbit. Experiment operations include inflight reconfiguration of the SDR waveform functions, and communications at S-band or Ka-band with NASA's inspace Tracking and Data Relay Satellite Network, or at Sband direct from the SCaN Tested to any Earth-based compatible ground station. One of the SDRs also receives GPS frequencies at the L1, L2c, and L5 frequencies. Various GPS waveforms are possible using the reconfigurable digital processing of the SDR. Command, control, and experiment operations occur from the Glenn Research Center in Cleveland, Ohio. NASA provides the ground connections between the Experiment Center and the uplink site to the TDRSS Network at White Sands. New Mexico.

Experimenters are encouraged to use the SCaN Testbed for a variety of research topics and applications. NASA will provide access and support to both the on-orbit testbed, and ground-based systems for development and verification activities. This paper will provide a description of the $\mathrm{SCaN}$ Testbed, the SDR capabilities, and the ground systems available to potential experimenters. Unique aspects of SDR developments, verification, and operations will be discussed.

\section{TABLE OF CONTENTS}

1. INTRODUCTION ............................................................ 2

2. FLIGHT SYSTEM................................................................ 3

3. GROUND SYSTEM............................................................... 3

4. FLIGHT SOFTWARE DEFINED RADIOS .......................... 4

5. AN EXPERIMENT ........................................................6 6

6. GROUND-BASED DEVELOPMENT SYSTEM........................ 7

7. FLIGHT SOFTWARE.................................................8 8

8. STRS REPOSITORY ......................................................8 8

9. SDR EXPERIMENT EXPERIENCE ....................................9 9

10. CONCLUSION........................................................ 10

REFERENCES................................................................... 11

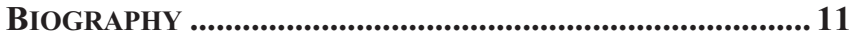




\section{INTRODUCTION}

The flexibility offered by software defined radios (SDRs) offer space missions new capability during the development and on-orbit phases to conduct and return more science. While the SDR provides this flexible capability for communications functions, navigation functions, and even networking functions of the mission, it also involves considering the way operations is viewed and conducted when using the SDR.

Software defined radios have emerged over the last decade as a viable approach to space communications. SDRs are capable of changing their characteristics through reprogrammable signal processing elements such as field programmable gate arrays (FPGA), or other technologies such as general purpose and multi-core processors. While SDRs have been considered or used in military domains [4], public safety [5], and cellular systems, SDRs are just beginning to emerge for space missions $[6,7]$.

Over the last 10-15 years, NASA has launched four missions and one demonstration carrying software defined radios. The Mars Reconnaissance Orbiter (MRO) [6], the Communication and Navigation Demonstration on Shuttle (CANDOS) [8], the Lunar Reconnaissance Orbiter (LRO) [7], and most recently the Curiosity (Mars Science Laboratory-MSL) and Mars Atmosphere and Volatile Evolution (MAVEN) each use an SDR as part of the mission. The MRO mission provides relay communications between Mars proximity surface rovers and Earth. Curiosity is a rover on the surface of Mars since 2012 and MAVEN is study the thinning of the Mars atmosphere and the disappearance of surface water over time.

MRO, MSL, and MAVEN all use a version of the Electra Ultra High Frequency (UHF) Transceiver. The Electra SDR is a fully reconfigurable, frequency-agile transceiver operating in the 390-450 MHz Ultra High Frequency (UHF) band. Future missions such as ESA Exobiology on Mars (ExoMars) and the Mars 2020 lander under development at Jet Propulsion Lab (JPL) are also planning to carry an Electra, as well as a newer Universal Space Transponder (UST) SDR which supports both X-band deep space communication and UHF proximity communications. The core of the Electra SDR is the baseband processor module (BPM), a flight-reprogrammable subsystem offering digitally implemented modulation and demodulation functions, standardized link layer protocols, and interfaces with the spacecraft avionics. This software defined radio BPM incorporates two key reconfigurable elements: a payload controller based on a SPARC 32-bit microprocessor and a modem processor utilizing a 1Mgate Xilinx reprogrammable Virtex FPGA. In addition, the baseband processor includes several radiation hard, one-time programmable FPGAs, along with a substantial amount of dynamic and static memory.

CANDOS was an SDR experiment run from the payload bay aboard a Space Shuttle flight. The experiment flew a Low Power Transceiver (LPT) SDR. The radio transmits and receives S-band bidirectional communications with TDRSS and receives GPS L1/L2 signals. Like other SDRs, the central part of the radio is the digital signal-processing module. The digital module hosted two Virtex II-6000 FPGAs, a Analog Devices DSP chip, memory for program storage, clock synthesis and distribution circuitry and various data transceivers.

The LRO mission was a lunar surface-mapping mission using a SDR for the radar waveforms. The LRO transceiver consists of analog receiver, digital receiver, quadrature digital waveform synthesizer, analog exciter, power amplifier, and control processor modules. The digital receiver and quadrature digital waveform synthesizer (QDWS) enable the flexibility and reprogrammability by implementing radar waveforms in Xilinx FPGAs. The Rad 750 control processor collects and reports telemetry to the spacecraft host, controls and configures the radio (and payload), and provides a router for radar data from the receiver to the spacecraft host electronics. The radio operates in the S-band and X-band.

To further expand the applicability of SDRs, reduce the perceived risk of using SDR technology, and understand SDR developments, operations, and performance in space; NASA has designed, built, launched, and installed a SDRbased communication system on the International Space Station, referred to as the Space Communication and Navigation (SCaN) Testbed.

The SCaN Testbed System consists of both a space flight element and ground control center used for operations. The SCaN Testbed provides a unique opportunity for researchers to investigate a host of different architectures and SDR applications in the environment of space using a complete end-to-end communication system. The use of SDRs as the radio platform of the SCaN Testbed provides a flexible and adaptable means to test a variety of communications, navigation, and networking protocols, and signaling schemes within an actual mission experience in the space environment. This unique opportunity is intended to expand the knowledgebase of SDR system users and provide mission managers with the information they need to reduce risk and cost to future science missions.

The goals of the project include demonstrating a seamless, interconnected, and autonomous end-to-end space network. The envisioned network will increase utilization over existing architectures, allows the flight system to perform functions currently performed on the ground such as scheduling link services and calculate spacecraft antenna pointing angles, adjust data rate, modulation, and error correction coding in response to channel conditions, automatically re-establish disrupted links, and route data according to priority and link conditions, among other new features. These new capabilities represent significant advancements in NASA's space networks. Enabling these 
advancements is the use of SDR technology. The SDR technology includes more than just the hardware platforms. SDR technology includes the platforms, software infrastructure, software applications, verification methods, and operations. Software infrastructure might include an operating environment to abstract the application software such as STRS [TBD] or for terrestrial military radios, the Software Communications Architecture (SCA) [TBD]. It might also include radio services such as temperature compensation circuits for receive or transmit functions, power amplifier control or protection, file services or other. Software applications also include more than typical modem functions such as modulation or encoding. These applications might include data formatting, link layer framing (e.g. using the Consultative Committee for Space Data Systems Advanced Orbiting System (AOS)), networking layers including Internet and disruptive tolerant networking protocols (IP and DTN). For integration and operations, the Testbed provides opportunity to better understand the activities associated with using SDRs. From new SDR software development, pre-flight verifications with ground hardware, software uploads to the flight system, and eventual over-the-air operations, use of the Testbed by NASA and outside experimenters is an allinclusive view of this important technology maturing to the state sufficient for routine use in space.

\section{FLight SySTEM}

The flight element is a fully space qualified communication system, pictured in Figure 1. The flight system consists of three software defined radios, each connected through an RF subsystem to the antennas. All subsystems are controlled from a flight computer referred to as the avionics. All the major elements of the flight system are housed within the main enclosure in the picture, with only the antennas visible.

Pictured in Figure 2, the flight element is installed on the truss of the International Space Station (ISS). From this location, the flight element communicates to NASA's White Sands Ground Complex via the Space Network of Tracking and Data Relay Satellite System (TDRSS), or direct communications with a ground station visible to the ISS orbit. Communications with TDRSS may occur at S-band or Ka-band frequency.

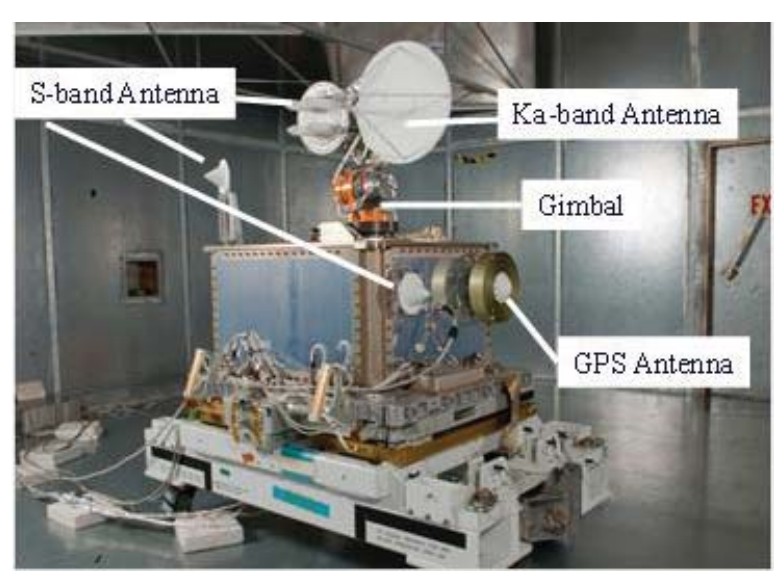

Figure 1 - SCaN Testbed Flight System

Communications direct with the ground occurs at S-band. The flight system also has a GPS antenna connected to one of the SDRs. The SDR can receive signals in the L-band, at and near the GPS frequencies of L1, L2, and the new L5. The remaining flight system components include the RF subsystem consisting of RF switches linking each S-band SDR to each S-band antenna. The Ka-band portion of the RF subsystem includes a Ka-band traveling wave tube amplfiier, and signal conditioning circuitry.

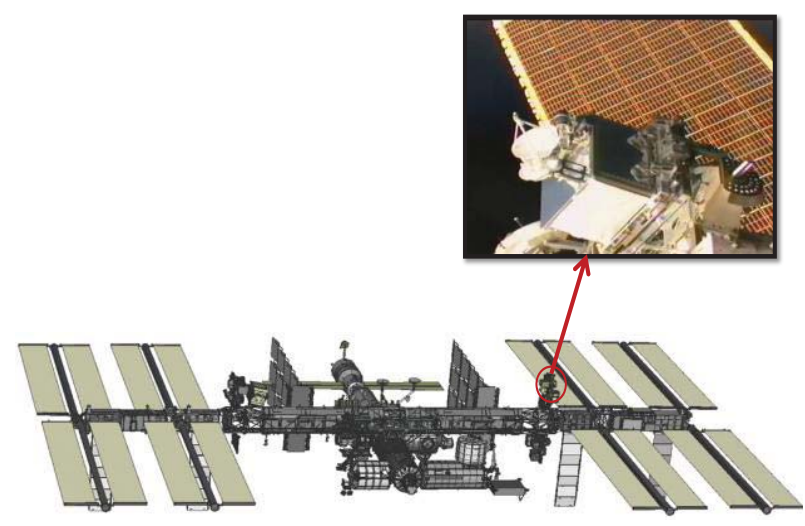

Figure 2 - SCaN Testbed Location on ISS

\section{GROUND SySTEM}

There are two paths to access the SCaN Testbed while onorbit on the ISS. The first path is NASA's ISS payloads' command and control path through the Payload Operations Integration Center (POIC) located at the Marshall Space Flight Center in Huntsville, Alabama. The path, shown in Figure 3 is from the SCaN TesBed Control Center to the payload through the POIC, is the primary command/ telemetry path to the TestBed. This path uses the ground infrastructure between POIC and WSC and the communication system on-board ISS established for all ISS experimenters to access experiment systems on-board ISS. 
The second path to communicate with the Testbed, is through the over-the-air experiment communications path from the experimenter to the software defined radios onboard the flight system. The experiment path is the main experimenter path to the SDRs, and illustrated in Figure 4.

Most experiments use the path through the SCaN Testbed Experiment Center (STEC) which provides a data interface to the TDRS and on to WSC. Some experiments may use experimenter provided communications terminals (e.g. a rooftop antenna at the experimenter location). The path from the Testbed to the Experiment Center begins at the Testbed from 1) within the SDR, 2) the avionics, or 3) incoming data on an experiment path routed back out on a second experiment path. From the SDR, the signal goes through the flight RF subsystem, through a user specified antenna (e.g. low gain or high gain) and communicates to a Tracking and Data Relay Satellite (within the Space Network) which serves as a "bent-pipe" relay satellite to a ground terminal at White Sands Complex or direct to a ground station. The White Sands Complex is one of the major downlink sites for satellites within NASA's Space Network System. The current operational configuration provides standard SN legacy services (modem, coding and other baseband bit handling), and a user programmable SDR is scheduled for installation at WSC. From the White Sands ground terminal, an experimenter may access either legacy services provided by the ground terminal (e.g. modem, baseband framing, network routing) or use a SCaN Tetsbed Project provided software defined radio to implement a custom application provided by the experimenter. The SCaN Testbed ground-based SDR provides the modem, baseband, and network routing functions for the experimental link, not supported by the legacy ground terminal services.

Once the data reaches the network routing stage, the experiment uses NASA's ground network to link the data to the SCaN Testbed Control Center and Experiment Center at Glenn Research Center. The Control Center routes experiment data to the Experiment Center for final reception and processing by experimenter provided hardware (as needed). At the Experiment Center, the experimenter also has access to operational information such as flight system telemetry, SDR telemetry, TDRS link parameters (e.g. BER, $\mathrm{C} / \mathrm{No}$ ), and ISS position and attitude information. The Control Center also provides a remotely accessible database of telemetry which can be retrieved by experimenters.

\section{Flight SoftWARE Defined RAdios}

Within the flight system are three software defined radios. The software defined radios each provide a different capability to the experimenter. One SDR built by General Dynamics Corporation, transmits and receives in NASA's near-Earth S-band allocation. The second SDR built by Jet Propulsion Laboratory and L3 Cincinnati Electronics, also supports transmit and receive at S-band and also receives $\mathrm{L}$ band GPS signals. The third SDR build by Harris Corporation, operates at the $22 / 26 \mathrm{GHz}$ Ka-band allocation.

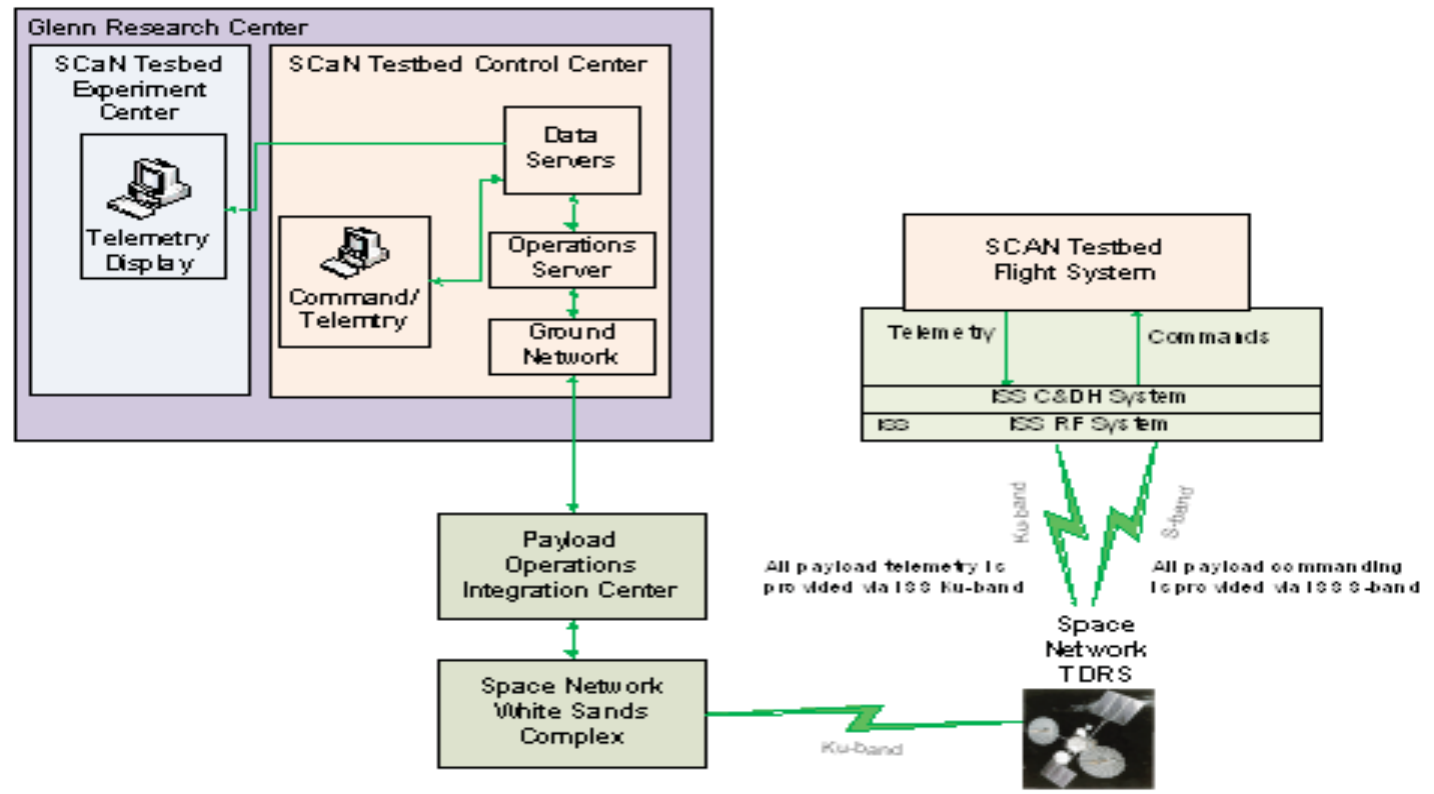

Figure 3 - Primary Command/Telemetry Path Ground System 


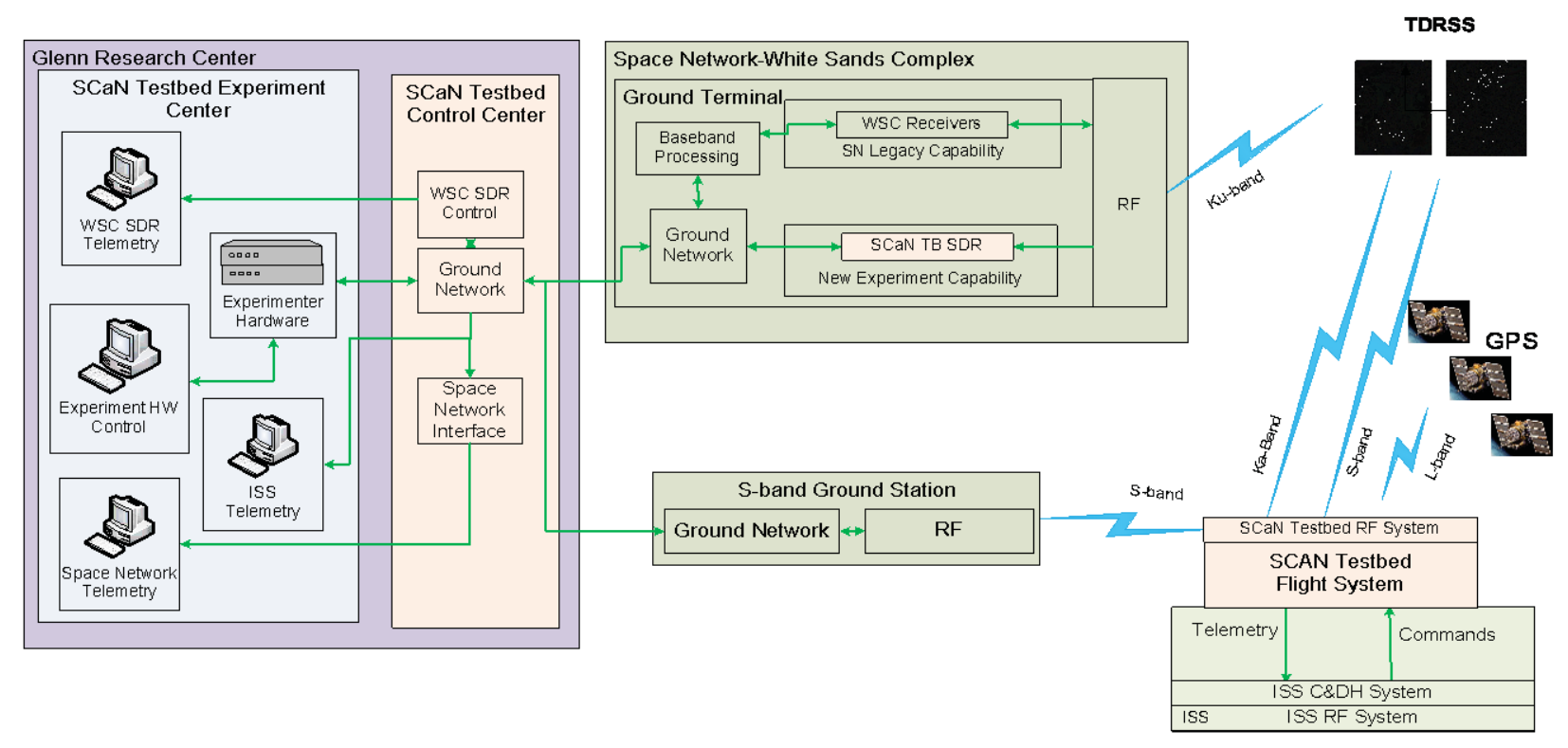

Figure 4 - Experiment Data Path Ground System

All three SDRs share a similar internal architecture. At the core of each SDR are a general purpose and signal processing module consisting of a general purpose processor, one or more reconfigurable FPGA's, and memory to store and run software, as well as (depending on the waveforms instantiated in the SDR) storing data that is being carried over the RF links. The remaining modules in each SDR include RF transition from the signal processing module to the power amplifier and receiver/low noise amplifier front end. Each SDR also interfaces to the payload avionics computer with both command/telemetry and data interfaces.

Each SDR is compliant to NASA's Space Telecommunications Radio System (STRS) [9]. Over the last several years, NASA has been developing STRS as a common architecture for SDRs. The STRS Architecture provides an abstraction between the radio platform hardware and the waveform applications. STRS allows NASA to successfully apply software defined radio technology in a common way across missions while preserving the unique implementations necessary to meet specific mission needs by developing radios that are based on an open architecture standard. An open architecture standard promotes the use of published, well defined interfaces that enable different vendors to provide radios that conform to the interface standard thus providing commonality among different implementations and enabling interoperability between providers of different software elements. Platform providers abstract hardware interfaces so as to work with waveform applications from different developers. Standard interfaces provide flexibility for component replacement, technology insertion, and risk reduction through standard software component reuse. Standards promote the growth of a large base of domain experts; agency personnel, software and hardware providers, and the user and operations communities, all knowledgeable of the common standard. NASA's goal in developing the STRS standard is to simultaneously capture the benefits of SDR technology and the economies and benefits of an open architecture standard.

Each Testbed SDR is compliant to the STRS Architecture, version 1.02.1. Each SDR provider delivered an operating environment, which included an abstraction layer for hardware interfaces and the required documentation for future waveform developers. In addition to the STRS compliant infrastructure platform software, GD and Harris also provided application waveforms to support an initial capability with Space Network Users Guide (SNUG) compatible modulation with their SDR, also compliant with the STRS Architecture. JPL provided a set of sample waveform applications to allow characterization and demonstration of the hardware platform, as well as an example for waveform developers. The Glenn Research Center teamed with Goddard Space Flight Center to provide a SNUG compatible waveform application for the JPL/CE SDR. This was the first time a waveform developer different from a platform developer provided a waveform, exercising the STRS Architecture interfaces and principles. While there were some dependent aspects on the particular abstraction infrastructure software, much of the waveform was developed in parallel with the radio which was enabled by the pre-defined STRS software interfaces. 


\section{AN EXPERIMENT}

An experiment using the SCaN Testbed can vary depending on the objectives. Experimenters may use existing capabilities of the Testbed in new ways, or provide new hardware or software for integration into the SCaN Testbed System.

Experimenters may develop experiments by adding software/firmware and/or hardware in various places within the SCAN Testbed System, illustrated in Figure 5. The overall system consists of the Testbed aboard ISS, the External Systems of White Sands or a direct to ground Earth station, the SCaN Testbed Ground System and finally the Experiment Center.

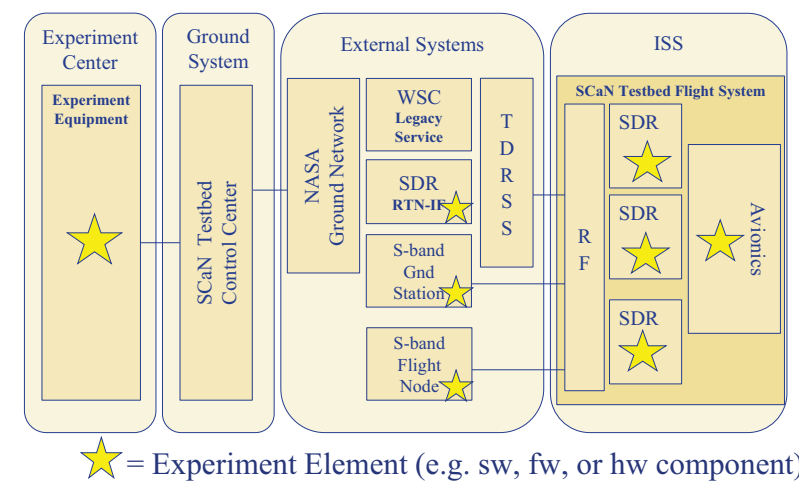

Figure 5 - Experimenter Access Points

Each star of Figure 5 represents locations where software functions or hardware may be provided by experimenters within the system. Software/firmware can be installed and operated on the flight system software defined radios and/or avionics. On the ground, software/firmware can be installed and operated on the SDR at the White Sands Complex.

An experiment could be a new waveform application installed on one of the SDRs communicating with the legacy services of the Space Network through a TDRS. This would entail the experimenter developing, testing, and providing the new firmware to the mission operations team for upload to the flight system. Alternatively, an experimenter could provide unique application software in the avionics, and use an existing waveform for the SDR link to the ground. Experimenters may also provide unique experiment hardware at either a ground station, at the SCaN Testbed Experiment Center, or even a separate flight node such as a cubesat and use SCaN Testbed as a relay satellite or another space node of the network. Hardware at the Experiment Center may send and receive directly with the data stream to the flight SDRs (via WSC) through the SCaN Testbed Control Center interface. Experimenters have the option to define their experiment and provide the hardware and/or software as they need for their experiment.
As an example, let's consider the SDR technology advancement experiments of the Glenn Research Center. The objective of these experiments is to advance the SDR platforms to Technology Readiness Level 7 (operation in the space environment, in a system configuration), characterize the launch waveform performance on-orbit, understand the operational aspects of multiple SDR waveform and application reconfigurations, and develop new platform services or applications to use the SDR to characterize the space environment effects on SDRs.

For these experiments, the experimenter provided the legacy TDRS waveforms that reside on each of the SDRs. Glenn Research Center and Goddard Space Flight Center teamed to develop the waveform on the JPL SDR, while Harris and GD provided the waveforms for their respective SDRs, to support the experiment. At WSC, the legacy receivers/modems are used for modulation and demodulation functions. In addition to the WSC provided hardware, the GRC experimenter provides a front end processor (EFEP), for higher layer functionality baseband processing (reference Figure 6). The EFEP creates (transmit side) and removes (receive side) a portion of the SDR waveform processing of the experiment data. The EFEP firmware can generate test data or read from files to provide a transmit bit stream through the SCaN Testbed system with a number of different bit patterns and at a variety of data rates. The experimenter controls the selection of waveform characteristics such as framing, randomization (or scrambling), and forward error correct (FEC) coding applied to the data if called for by the experiment configuration. The EFEP can also receive experiment data from the SCaN Testbed system and remove these waveform characteristics to restore the user data. In this example, the experimenter provided both the hardware and software for the experiment [12].

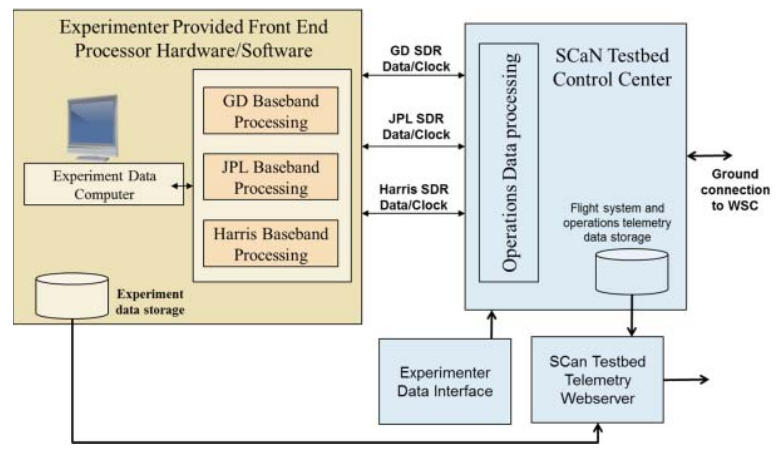

Figure 6 -Experiment

Hardware/Software Example

Figure 6 illustrates a functional diagram of the experimenter provided front end processor and the clock and data interface between the experimenter provided hardware and the SCaN Testbed Control Center. The experiment hardware is also equipped to accommodate commercial bit error rate test equipment, data storage, and a network interface. This example experiment hardware is expected to be more extensive than typically provided hardware since 
the Glenn Research Center will reuse the hardware for a number of different experiments.

Other early experiments include an S-band and Ka-band IPv4 On-board Routing/Relay Experiment to demonstrate IP routing between a ground and on-board network using one or more $\mathrm{Ka} / \mathrm{S}$-band point-to-point sub-networks formed by TDRSS legacy waveform and IP gateways available at launch. This experiment will demonstrate application data routing between ground hosted and on-board hosted applications over various IPv4 sub-networks. The IP applications are isolated from data link changes by the IP layer, thus various waveforms with different data rates or data format characteristics can be used, such as those provided by the launch waveform experiment EFEP, shown in Figure 6. The routing/relay applications do not have to be modified as new SDR waveforms or better data links are introduced (IP on radio).

Another early experiment is GPS navigation at the L1, L2, and L5 frequencies. GPS L1 signals have been used by LEO satellite systems for navigation for a number of years. The SCaN Testbed adds the new L5 and L2c signals and provides a unique capability to modify on-orbit algorithms and assess performance at L1, L2, and L5. This particular GPS experimenter, the Jet Propulsion Laboratory, has developed the software (both CPU and FPGA code) required to receive L-Band GPS signals on the JPL SCaN Testbed SDR, to provide real-time precise positioning of the GPS antenna on the SCaN Testbed and compare with the known ISS position solution. As new GPS services are enabled, new GPS waveforms can be implemented and verified on the flight system by any experimenter. This GPS experiment will also use ground processing to determine the ISS orbit, calibrate the ionosphere effects using the at least two GPS frequencies and characterize the multipath environment at the GPS antenna on the SCaN Testbed.

\section{GROUND-BASED DEVELOPMENT SYSTEM}

Once an experimenter's proposal is submitted, reviewed and selected by the Experiment Board, experimenters will have access to several SCaN Testbed project ground based systems for development and verification of their experiment software. GRC and JPL provide testbeds with radio prototypes or breadboards. There is also a high fidelity replica of the entire flight system at GRC containing Engineering Model (EM) copies of the flight articles, referred to as the Ground Integration Unit (GIU).

The GRC Experiment Development System (EDS) is a collection of SDR breadboards and associated test equipment intended to provide initial opportunity for software testing and basic functional validation. The EDS equipment provides a basic emulation of the hardware and software of the SCaN Testbed flight system. Configuration control of the EDS is not as formal as the GIU and thus affords more of the laboratory development work environment.

Experimenters may access the EDS physically at NASA's Glenn Research Center or through a remote connection. In person use of the SDR breadboards may provide additional test points depending on the final remote access design. Remote access to the breadboards will save travel time and expenses for experimenters. In either case, project personnel will assist experimenters in the lab or using the remote connection to the hardware. JPL also provides a remotely accessible prototype testbed for the JPL-SDR. An important difference between the JPL testbed and the GRC EDS is that there is no avionics at JPL, so experimenters are working directly with the radio's command, telemetry, and data interfaces.

The GIU is a high fidelity version of the SCaN Testbed flight system. The GIU is used for both experiment software development and for more controlled final development testing and verification testing. Because of the high fidelity of the GIU, it is also used by the SCaN Testbed Mission Operations team for mission training and flight operations anomaly resolution. Due to the limited EDS capability, in some cases the experimenter may initiate ground test exercises on the GIU and corresponding support systems (i.e. bypassing using the EDS capabilities). Figure 7 illustrates the development process [10].

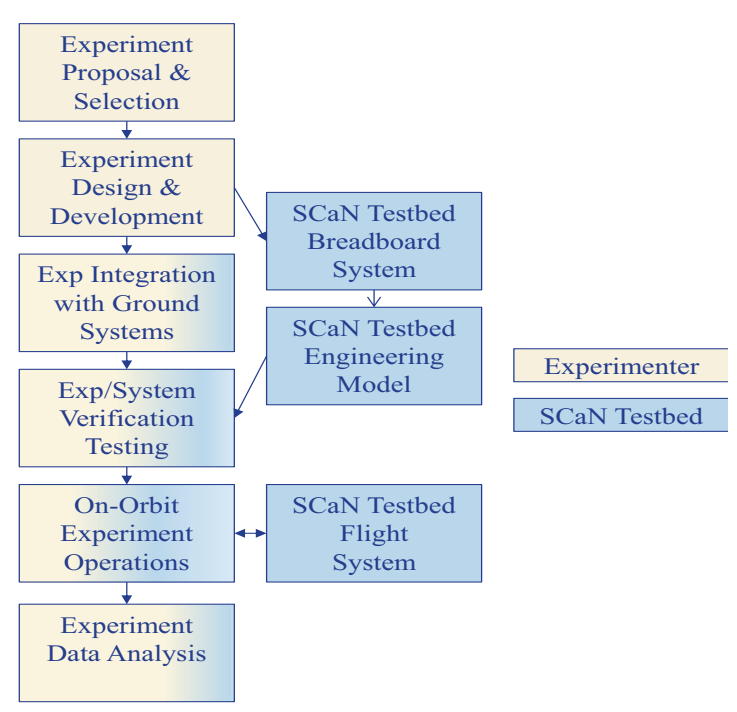

Figure 7 - Experiment Development Process

Experimenters may access the GIU physically at Glenn. At this time there is not a remote connection planned to the GIU. Project personnel will assist experimenters on-site using the GIU.

Generally, experimenters will develop and operate their experiment with no cost to NASA. Experimenters have access to NASA personnel, ground development systems, 
and the flight system. However, experimenters may request from NASA additional, specific support for their experiment which may result in reimbursement to NASA, but this is a unique situation determined at the start of the experiment discussion. Experimenters will be allotted time and support from the project depending upon the number of experimenters and available project resources. Every effort will be made to accommodate experimenter's time with the system.

\section{FLIGHT SOFTWARE}

Software developed for the Testbed is subject to the NASA Procedural Requirements (NPR) Software Engineering Requirements 7150.2 [11] processes and procedures for developing space flight software. This entails creating certain plans, documenting requirements and designs, verifying operations, and performing certain code and project reviews. Experimenters are expected to conform to the intent of the NASA standards, but may use their own processes, if they align with the standard. The experiment plan will document the differences. An essential distinction in the level of process rigor is the NASA software class, which is different for software that will run on the avionics and that which will run on the SDRs.

Software can be placed on either the avionics or software defined radios. Software on the avionics performs a number of functions for experiments in addition to safety critical functions (e.g. applying power to amplifiers). The avionics software, including experiment software running on the avionics is all considered Class " $\mathrm{C}$ ", as defined by NASA [11]. The Class $C$ software process entails areas of planning, management, design, coding, testing, release, maintenance, assurance, and configuration management, and monitoring. While these guidelines attempt to be comprehensive to aid development and preserve safety of the equipment, NASA will work with experimenters to keep their development costs to a minimum. In many instances, standard company development practices satisfy NASA's development process requirements.

Software for the SDRs is considered Class "E+". This designation is a slight modification of the lowest classification for mission software, Class E. This designation is used for software where the consequence of the software failing to meet its objective or operate correctly only affects the experiment outcome, but poses little risk to the system itself. Software issues within the SDRs are generally contained within the SDR and do not pose a significant risk to the other parts of the system, unlike the avionics. This lower classification eases the development burden and in these instances, good engineering software development practices will generally comply with the requirements.

\section{STRS REPOSITORY}

Software defined radios present a unique opportunity to develop applications that have long term value for NASA. A repository will accept software artifacts (e.g. VHDL, XML, and documentation) developed for experiments, to enable future experimenters access to past applications, according to the STRS Architecture. The STRS Repository retains software, documents, and ultimately the application information so that the application knowledge may be passed from one mission to another as missions change. Any artifacts and documentation supporting the waveform application, including source code, models and algorithms, process flow diagrams, and documentation (e.g. design, test) are an integral part of the repository. Contributions may include proprietary content, or third party software components (e.g. IP cores for FPGA), so the repository documents the licensing and other usage restrictions. The goal is to build a repository of waveforms for NASA's use, while protecting the products and innovation of the developers. Figure 8 illustrates the concept of waveform submissions.

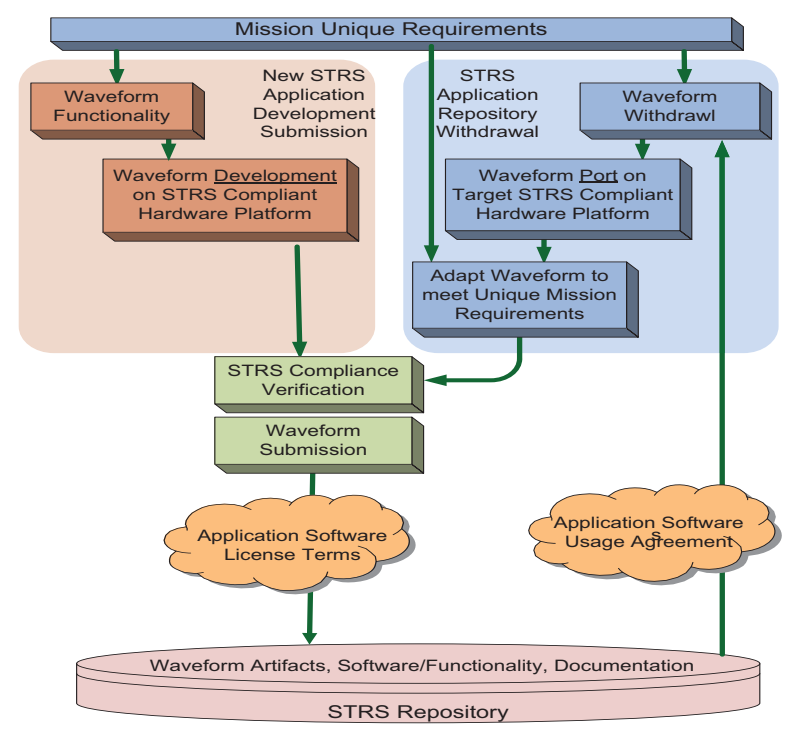

Figure 8 - STRS Repository Concept

The Glenn Research Center verifies that platform software and waveform applications comply with the STRS architecture and certify the modules submitted to the library for future reuse. The STRS architecture will evolve through appropriate change process and controls to ensure that the architecture continues to meet evolving mission needs and allows mission to infuse new technology.

Individual missions will apply the STRS architecture to meet specific requirements and radio development needs. Mission designers will determine the implementation approach for a particular platform by specifying the particular type of radio required, with the set of features and operating functions needed to accomplish the mission. The missions will tailor the architecture for the specific radio use 
and implementation while remaining compliant with the architecture. The library of software modules provides mission designers a starting point for waveform applications. Missions will both submit their waveforms to the repository or use waveforms from the repository. Designers could adapt modules used from the library to meet individual requirements and needs reducing their project costs. Waveforms used from the repository will be shared with appropriate use agreements to protect he original developers. Designers will also provide valuable feedback to improve the architecture definition for uses and accommodate current or available technology.

Communication radio providers within the space products industry will develop architecture compliant radios to meet architecture \& mission requirements through traditional acquisition procedures. The repository offers an opportunity to reduce develop costs by allowing developers to reuse existing software from NASA's STRS repository and utilize elements of compliant designs over multiple missions to reduce radio costs.

\section{SDR EXPERIMENT EXPERIENCE}

Having SDRs in space offers many opportunities for test and evaluation of new concepts both for the SDR applications and signaling and with the operational use of SDR technology. A few of the lessons associated with the SDR technology itself include: the SDRs require personnel with a wide skill set, SDR flexibility leads to complexity, software management includes both space and ground assets, characterize SDR platform along with applications, and provide a flexible method to add commands and telemetry.

SDR personnel skill set: SDRs are small and compact, yet they are complete communication systems. For hardware, they often include digital signal processing, up and down frequency conversion, transmit or receive RF sampling, stable oscillator subsystems, and RF amplifiers. In software they include many functions beyond just the "modem". They now include the software infrastructure (such as STRS), radio services such as power amplifier monitoring, temperature control or protection, file management, and others. Applications include traditional modem functions such as modulation and encoding, but could also include many more software functions such as data framing, network routing (e.g. Internet Protocol (IP) or Disruptive Tolerant Networking (DTN), and applications using these different capabilities. In addition, many of NASA's services conform to the Consultative Committee for Space Data Standards (CCSDS), thus requiring additional skills. While it's possible to have the software engineer only worry about the infrastructure, they cannot discount what the waveform application might require from the platform operating environment. The waveform developer must understand the RF portions of the platform, to better understand their own requirements and needed performance along with adherence to any applicable standards. And finally, the system engineer needs to oversee the entire link end-to-end, with much of the space link processing (in this case) within the SDR itself.

SDR flexibility leads to complexity: While a mission may never reconfigure its complete function everyday like the SCaN Testbed for obvious reasons, SDRs still provide a lot of flexibility which could lead to considerable complexity if not managed properly. The entire process of developing new software requires a ground unit SDR mostly identical to the flight unit. The SCaN Testbed engineering models generally used flight parts (for functionality) but with less screening (for cost) than the flight radio. Even with these measures, an early waveform still experienced peculiar onorbit behavior over temperature, not experienced on the engineering model. While the tolerances for temperature were designed for the on-orbit ranges, adjustments still had to be made. At issue seemed to be how the FPGAs were instantiated upon waveform load. Modifying the load software along with verifying the instantiation helped resolve the problem.

Software management: The software for both development of new waveforms (i.e. development tools) and the on-orbit waveform or infrastructure software must be carefully managed. Software development tools (and sometime the development hardware itself) go obsolete long before the space hardware fails. This creates issues to have compatible development boards and tool chains for new waveform development. The array of developers (e.g. infrastructure, applications, application integrators) provide multiple versions of different software, sometime for each new waveform. It's imperative to use good configuration practices, for both ground and flight, and be able to manage all combinations (e.g. OE 1 and WF 1, OE2 and WF2, OE2 and WF1, etc. especially when the OE is part of a waveform build). Due to on-orbit anomalies, the project had circumstances where it used older versions of infrastructure software, but with a newer waveform. This creates an issue if one wants to verify by test every combination on the ground, versus doing verification by analysis or inspection to reduce time/cost to get something on-orbit.

One way to manage or reduce the complexity with SDRs is with new automated functions or adding cognition within the SDR to offload operations. The SCaN Testbed plans to develop a number of applications (both flight and ground) to look at how these new functions such as cognition could be useful in a space mission, and what functions make sense to perform with the radio. Link management, data management, and in the future, spectrum management will all be investigated.

Characterize SDR platform along with applications: Platform hardware is generally much more capable than what initial waveforms might use initially during a mission. SCaN Testbed initial waveforms were compatible with the 
existing services at Space Network (NASA's space infrastructure), but the project envisioned advancing new waveforms for future uses. The requirements for future capability must be flowed down to the platform developer and the system developer in such a way to document the launch capability and understand the performance of the radio in the system. Requirements must reflect platform capability (not just initial waveforms) for verifications. Platform providers could also provide test waveforms for the verifications. Having waveform tools for such tasks as recording raw $\mathrm{ADC}$ samples for post processing (variable sample rates, durations, etc), transmitting arbitrary signals or perhaps data from a stored file provide significant capability once the radio is integrated into the system. These tools alone provide transmit and receive capability to test the entire system without using a specific modulation and coding combination that might be used for the mission. In addition, lower level radio services (non-waveform software) to measure receiver gain, receive power level, transmit power level (may require hardware sensor), and frequency tuning are all key items to control or measure.

Flexible method to add commands and telemetry: Because SDRs are reprogrammable on-orbit, there needs to be an architecture developed at requirements formulation time to add new waveforms and provide a path for the associated new commands and telemetry. These requirements may have impact on the platform developers. The operations centers for space SDRs will originate the commands and display telemetry of the SDRs. In the STRS model, new waveforms could come from a third party developer who needs the platform to deliver their telemetry to the ground and they may require new commands be added to the platform. Due to the compressed schedule, the SCaN Testbed accepted the command/telemetry interface from each platform developer "as is", and it provides an opportunity to see three different approaches. The GD SDR uses a very efficient, but more stringent command and telemetry interface. Bit positions within 1553 structure indicate operational modes and telemetry measurements. Changing waveforms on-orbit entails changing the 1553 bit structure and definition on the radio and within the avionics (flight computer). The Harris SDR uses a name value pair defined by the waveform application. This provides more flexibility for new waveforms, but also introduces configuration files to define the name and value pairs. The JPL SDR infrastructure provides a few words of telemetry (sent to the ground periodically) for new waveforms but relies more on a serial console looking interface providing text over 1553, with variable size and rate, and a free format. While it is most flexible, this now creates a file on the ground which is saved separate from the spacecraft telemetry data. Thus in all these options, the command and telemetry architecture needs to consider the new waveform telemetry, how to bring the data down to the ground (with spacecraft telemetry or separately), how to store the telemetry (filed with spacecraft data or separate), flexibility to add telemetry or commands, including updates to the command dictionary (both on-board and documentation), and overall data management.

On the ground, for all the radios, screens display telemetry from the launch waveforms. New waveform telemetry generally requires new screens be developed within the ground software to display new telemetry. In some circumstances, new raw telemetry may reuse existing screens, but will lack any engineering conversion on the ground before display. To minimize changes on the ground, an architecture that uses perhaps name value pairs, and then builds screens based on the new configuration will reduce development time and costs for new waveform updates.

\section{CONCLUSION}

NASA's SCaN Testbed is a flight communication system installed on the external truss of the International Space Station. The flight system consists of three software defined radios capable of communicating with the Tracking Data Relay Satellites of NASA's Space Network (GEO satellite infrastructure), or directly with ground stations located anywhere on the Earth within view of ISS. The system can communicate at the S-band or Ka-band frequency. The system also has an SDR that receives at the L-band frequency to receive GPS or other global navigation satellite system signals.

The unique aspects of the SCaN Testbed allow investigations in the environment of space, using fully space qualified, radiation tolerant electronics. All aspects of both the flight and ground system are implemented and available.

The SCaN Testbed Project is studying the application of SDRs to NASA missions, through experiment use of the Testbed. Experimenters from NASA, industry, academia, and others are invited to use the Testbed to conduct experiments in communication, navigation, and networking applications. The experiment applications on the SDRs are compliant to NASA STRS Architecture, a common architecture for SDR platforms and applications to enable waveform abstraction from underlying hardware. The enduring nature of software and the common STRS Architecture provide a repository of applications to enable software reuse among missions employing STRS compliant SDR platforms. The SCaN Testbed, and STRS are paving the future for NASA's use and understanding of software defined radios for space.

Lessons learned from operating the SDRs include understanding the skill set required for developing and operating SDRs, which exceed traditional communication radios, understanding how to manage SDR flexibility such that it does not lead to additional complexity and cost, characterizing the SDR platform along with applications, and providing a flexible method to add commands and telemetry 


\section{REFERENCES}

[1] D. Grillo, P. Valigi, "A Testbed for Assessing the Traffic Performance of Adaptive", Personal Communications Gateway to the $21^{\text {st }}$ Century, Conference, 1993.

[2] R. Chertov, D. Havey, K. Almeroth, "MSET: A Mobility Satellite Emulation Testbed", INFOCOM, Proceedings IEEE, March 2010.

[3] J. Lietner, "A Hardware-in-the-Loop Testbed for Spacecraft Formation Flying Applications", IEEE Aerospace Conference, March 2001.

[4] K. Richardson, C. Jimenez, D. Stephens, "The Evolution of the Software Comunications Architecture", IEEE Military Communications Conference, October 2009.

[5] A. Gorcin, H. Arslan, "Public Safety and Emergency Case Communications: Opportunities from the Aspect of Cognitive Radio" IEEE Symposium on New Frontiers in Dynamic Spectrum Access Networks, 2008.

[6] C.D. Edwards, et al., "The Electra Proximity Link Payload for Mars Relay Telecommunications and Navigation", 54th International Astronautical Conference, Bremen, Germany, 29 Sep 2003.

[7] K.R. Raney, Life Fellow IEEE, P.D. Spudis, B. Bussey, J. Crusan, J. R. Jensen, Member IEEE, W. Marinelli, et. al. "The Lunar Mini-RF Radars: Hybrid Polarimetric Architecture and Initial Results" Proceedings of the IEEE. Volume: 99, Issue: 5, Page(s): 808-823, 2011

[8] S. Pace, et al., "Test Results from the Communications and Navigation Demonstration on Shuttle (CANDOS) Experiment", Intl. Symp on GPS/GNSS, Tokyo Japan, Nov 15-18, 20034.

[9] R. Reinhart, T. Kacpura, L. Handler, C. Hall, D. Mortensen, S. Johnson, et.al. Space Telecommunications Radio System (STRS) Architecture Standard, Release 1.02.1. NASA Technical Memorandum 2010-216809, December 2010.

[10] Space Communications Testbed Project, Experimenters Handbook, http://spaceflightsystems.grc.nasa.gov/SOPO/ SCO/SCaNTestbed/. 2012.

[11] NASA Procedural Requirement, 7150.2A, NASA Software Engineering Requirements, 2009.

[12] M. Shalkhauser, N. Adam, SCaN Testbed Project, Experiment Front End Processor Design. April 2011.

\section{BIOGRAPHY}

Richard C. Reinhart is a senior communications technologist with NASA's Glenn Research Center (GRC), located in Cleveland, Ohio. He currently serves as the Principal Investigator for NASA's software defined radio $(S D R)$ flight experiment aboard International Space Station, called the SCAN Testbed - Space Communications and Navigation Testbed. Mr. Reinhart has 25 years of experience with NASA's space communications technology development. His activities include satellite, ground station, high data rate communications, phased array antennas, and software defined radio technology, including development and a Principal Investigator of the Space Telecommunications Radio System (STRS) Architecture, flying for the first time in space in the SCaN Testbed SDRs. Mr. Reinhart has a number of technical papers and conference presentations on work associated with the SCaN Testbed (also under the title CoNNeCT, Communications Navigation, and Networking reConfigurable Testbed), the STRS SDR Architecture, and the Advanced Communication Technology Satellite (ACTS) Project.

James P. Lux is currently a Co-Principal Investigator for the SCan Testbed/CoNNeCT project. He is the Manager responsible for delivering a NASA STRS Operating Environment (the software infrastructure) for the JPL-SDR to the SCaN Testbed project. Previous work at JPL has included an adaptive phased array antenna with distributed processing and metrology, a ground station for on-orbit calibration of radar, and a variety of other radio and communications projects. Before joining JPL in 1999, he provided engineering consulting services to a variety of clients, including development of Electronic Warfare systems for broadband signals analysis and identification, jamming, and other countermeasures, large scale software database systems, and motion picture special effects. 